\title{
Correct Placement and Rapid Confirmation of Double Lumen Tube and Bronchial Blocker Using Fluoroscopic Guidance
}

\author{
Swati Deepak Parmar ${ }^{1}$, Avinash Sahebarav Kakde ${ }^{1 *}$, and Harshal Wagh ${ }^{2}$ \\ ${ }^{1}$ Clinical Assistant, Anaesthesia Department, Kokilaben Dhirubhai Ambani Hospital, India \\ ${ }^{2}$ Anaesthesia Consultant, Anaesthesia Department, Kokilaben Dhirubhai Ambani Hospital, India
}

"Corresponding author: Kakde AS, Clinical Assistant, Anaesthesia Department, Kokilaben Dhirubhai Ambani Hospital, Mumbai, India, Tel: +91-9664027829; E-mail: avinashkakde87@gmail.com

Received: August 23, 2018; Accepted: August 28, 2018; Published: August 31, 2018

Double Lumen Tube (DLT) and Bronchial Blocker (BB) are frequently used to provide One Lung Ventilation (OLV) in major thoraco-abdominal and spine surgeries. With advanced surgeries like Video Assisted Thoracic Surgeries (VATS), rapid and accurate placement of the DLT/Bronchial Blocker is necessary. Clinical examination includes inspection of chest movements and auscultation of breath sounds. These clinical methods remain important means of accurate placement of DLT/Bronchial blocker as a first confirmation test. However, these methods are subjective, and variability exists between individuals who are very common, and it fails to correctly identify the placement in $37 \%$ of the patients and requires repositioning by bronchoscopy [1].

Here, we describe the placement of DLT and Bronchial Blocker under fluoroscopic guidance. In our case, 50 years old male posted for open thoracotomy; had difficult intubation due to restricted mouth opening. He was operated for Carcinoma of right buccal mucosa and was posted for right upper lobectomy for metastasis. Awake nasal endotracheal intubation was planned using adult bronchoscope. Due to unavailability of pediatric bronchoscope, placement of blocker was planned under fluoroscopy.

The placement was done in real time in span of 20-30 sec (FIG. 1) and was also confirmed clinically. Repeat fluoroscopy was done after lateral position. Adequate collapse of lung was achieved. Same method was used for correct placement of DLT (FIG. 2) in other patients. Similarly, we did successful placement of DLT/Bronchial Blocker in 30 cases of VATS esophagectomy and lobectomy. Fluoroscopy is a noninvasive method with short learning curve. It emits low dose radiation and requires only 20-30 sec for confirmation. It is easily available in operating area and an ideal tool in centers where bronchoscopes, especially pediatric bronchoscopes are unavailable. In a center, where many thoracic surgeries and head-neck oncology surgeries are being performed, the fiber optic cleaning and sterilization is time consuming. Also, fluoroscopy helps to overcome the limitations of fiber optic bronchoscope e.g. unfavorable anatomy, bleeding during intubation and time

Citation: Parmar SD, Kakde AS, Wagh H. Correct Placement and Rapid Confirmation of Double Lumen Tube and Bronchial Blocker Using Fluoroscopic Guidance. Clin Case Rep Open Access. 2018;1(1):104.

(C)2018 Yumed Text. 
constraints. Fluoroscopy can identify wrong side placement of device without wasting much time. In medicolegal dispute we can easily produce print of fluoroscopic images.

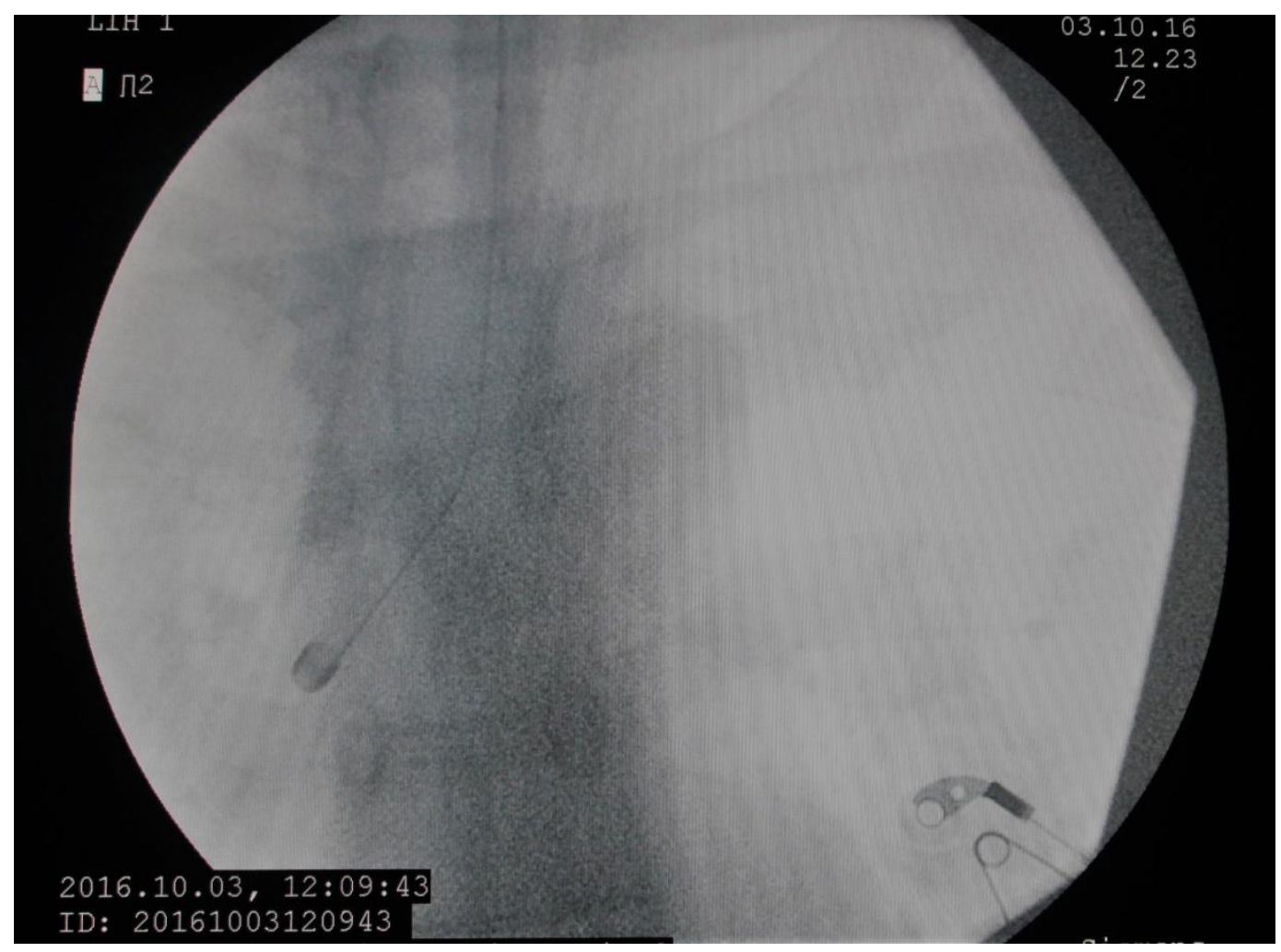

FIG. 1. Fluoroscopy showing correct placement of Bronchial blocker.

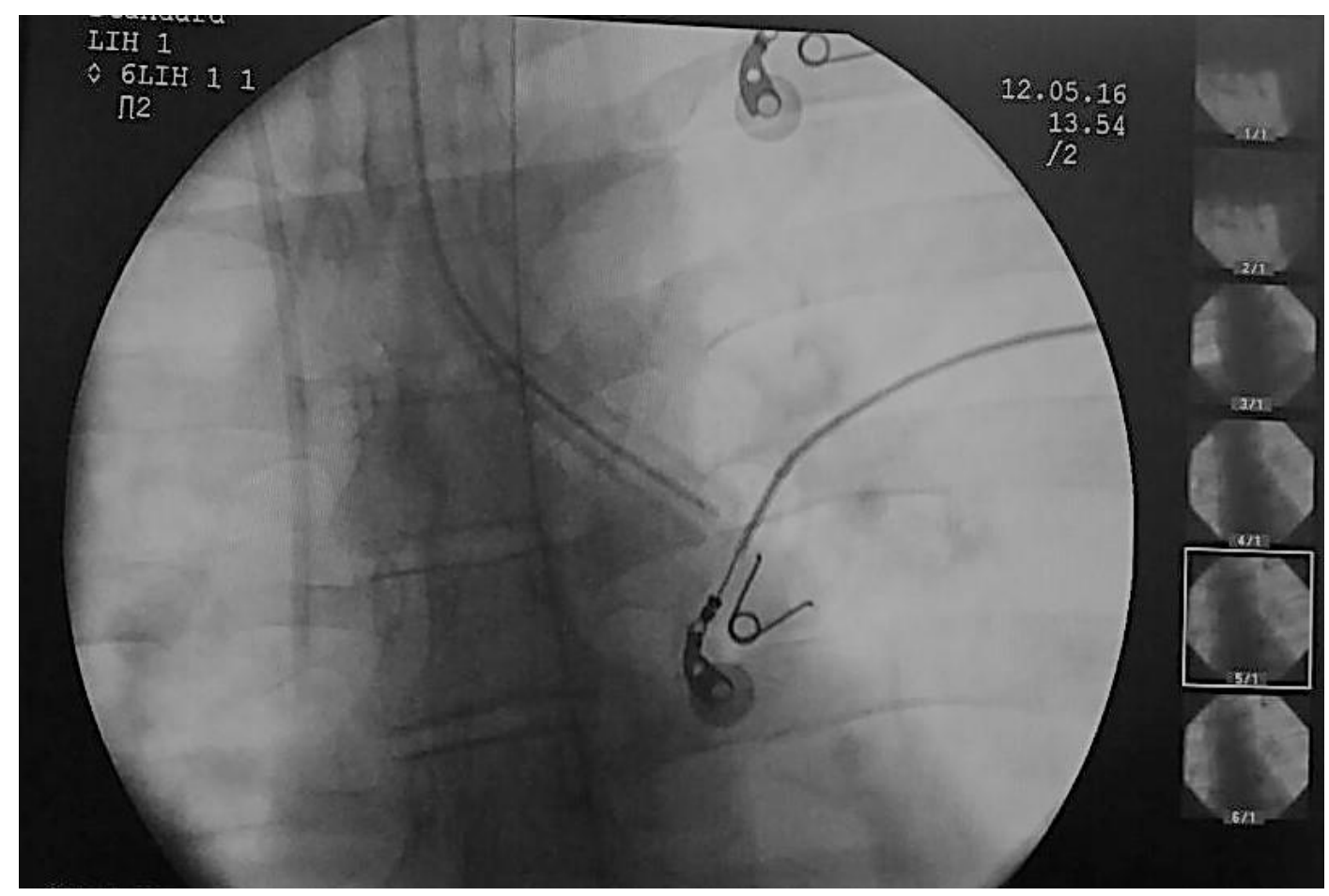

FIG. 2. Fluoroscopy showing correct placement of Double lumen tube. 
www.yumedtext.com | August-2018

As per the study done by Emile C, Jean M, et al. [2] also supports the use of fluoroscopy. In this study after intubation with double lumen tube first auscultation is done and irrespective of the findings fluoroscopy was used to confirm the same. They also mention the patient dose summary of radiation. The DLT was perfectly placed in $99.5 \%$ of patient using fluoroscopy [2].

Fluoroscopy is an invaluable tool in very young pediatric patients for confirming lung isolation as pediatric bronchoscope (3 $\mathrm{mm}$ ) cannot be used for pediatric patients less than 2 years of age [3]. Few studies also emphasize importance of using USG [4].Fluoroscopy has many other advantages when compared with Ultrasonography (USG). It can detect lung inflation and deflation in real time and can compare bilaterally. It can also visualize lung in cases of lung pathologies. High interindividual variability exists amongst users of USG and requires expertise for the same. There are few centers where USG machine is not easily accessible/available. In case of right sided DLT, fluoroscopy can visualize apical lobe ventilation and DLT tip placement which is difficult to visualize only with the help of USG.

So, to conclude fluoroscopy is an acceptable, easily available, low cost and reliable technique to confirm the lung isolation in adults as well as in pediatric patients.

\section{Conflict of Interest}

No conflict of interest.

\section{REFERENCES}

1. De Bellis M, Accardo R, Di Maio M, et al. Is flexible bronchoscopy necessary to confirm the position of double lumen tubes before thoracic surgery? Eur J Cardiothorac Surg 2011;40(4):912-6.

2. Emile $\mathrm{C}$, Jean MB, Ridha H, et al. Fluoroscopic guidance for placing a double lumen endotracheal tube in adults. Acta Anaesthesiol Taiwan. 2014;52(3):107-9.

3. Letal M, Theam M. Paediatric lung isolation. BJA Education. 2017;17(2):57-62.

4. Parab SY, Divatia JV, Chogale A. A prospective comparative study to evaluate the utility of lung ultrasonography to improve the efficacy of traditional clinical methods to confirm position of left sided double lumen tube in elective thoracic surgeries. Indian J Anaesth. 2015;59(8):476-81. 